\title{
Applications of Magnetochemistry to Polymers and Polymerization ${ }^{1}$
}

\begin{abstract}
By Pierce W. Selwood ${ }^{2}$
After a brief review of fundamental definitions, experimental methods, theoretical and semiempirical results on diamagnetic substances, two topics are considered: First, studies of the diamagnetic anisotropy of crystalline and oriented materials. Using solid naphthalene as an illustration, it is shown how to obtain the principal molecular susceptibilities from the measured parameters of the single crystal and the molecular orientations in the unit cell as derived from X-ray studies. Conversely, the direction cosines for solid diphenyl are calculated from the measured macroscopic susceptibilities and the susceptibilities of the molecule. Results recently obtained on the anisotropy of cellulose, protein fibers, and stretched rubber indicate the value of such methods, particularly for oriented polymers containing aromatic groups, because of the large effects of the latter. Next, attention is devoted to the paramagnetism of oxygen and free radicals that can be utilized as a measure of the concentration of the species. Radical concentrations as low as $10^{-6}$ mole per liter may be estimated by means of a modification of the Gouy-balance. It has been employed in a study of the thermal polymerization of styrene at $66^{\circ} \mathrm{C}$. From the changes in diamagnetism in the course of the reaction, the rate of consumption of oxygen present is determined. Assuming this to be due to combination with styrene radicals, the rate of thermal initiation is estimated to be $3.8 \times 10^{-14}$ mole $^{-1}$ liter sec-1. However, in order to obtain by magnetic methods directly the free radical concentration in ordinary chain polymerization processes, the sensitivity would have to be improved by several orders of magnitude.
\end{abstract}

\section{Introduction}

There are two principal types of magnetic measurements from which polymer chemistry may gain information. These are (1) the study of diamagnetic anisotropy, and (2) the in situ study of free radicals and other paramagnetic components, such as oxygen. Neither of these methods has so far received much attention from polymer chemistry. There is reason to believe, however, that they will both make important contributions to our knowledge of polymers and polymerization in the not too distant future.

The numerical quantity with which magnetochemists are chiefly concerned is the magnetic susceptibility, $\chi$, This quantity is defined as follows: Let $H$ be the intensity of a magnetic field and $B$ be the magnetic induction in any

\footnotetext{
1 This paper was presented as part of the 1946-47 series of lectures on the Properties of High Polymers given at the National Bureau of Standards.

${ }_{2}$ Professor of chemistry, Northwestern University.
}

substance placed in the field. We then have $B=H+4 \pi$, where $\partial$ is the intensity of magnetization, and $\partial / H$ is the magnetic susceptibility per unit volume, $K$. Taking $d$ as the density of the substance: $\mathrm{K} / d=\chi$, which is the magnetic susceptibility per unit mass.

For nearly all organic compounds the magnetic susceptibility is negative. Such substances are said to be diamagnetic; they are repelled by a magnet. The order of magnitude of the susceptibility for organic compounds is $-0.3 \times 10^{-6}$ to $-0.8 \times 10^{-8}$. The susceptibility of water is $-0.720 \times 10^{-6}$. For diamagnetic substances the susceptibility is independent of field strength and virtually independent of temperature.

For many transition group elements and their compounds, and for organic and inorganic free radicals, the magnetic susceptibility is positive. These are said to be paramagnetic. The susceptibility of paramagnetic sulustances is often 10 to 
100 times as large numerically as that of diamagnetic substances. Paramagnetic substances are attracted to a magnet. Positive magnetic susceptibilities are substantially independent of field strength, but they frequently vary approximately inversely as the absolute temperature. More often, they follow the Curie-Weiss law, $\chi=C /$ $(T+\Delta)$, where $C$ and $\Delta$ are constants and $T$ is the absolute temperature.

Magnetic susceptibilities are most frequently measured by the method of Gouy (fig. 1). A

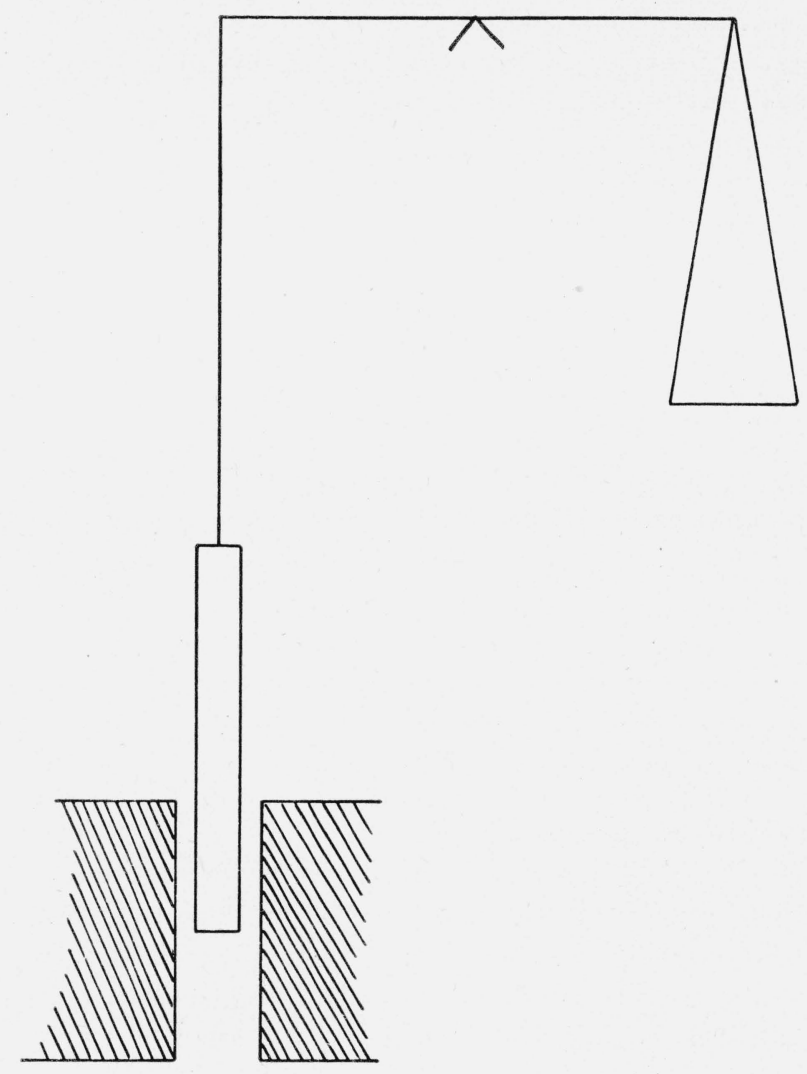

Figure 1. Principle of the Gouy method.

cylindrical sample is suspended from a balance so that one end of the sample is in a region of high magnetic field intensity, and the other end is in a field of negligible intensity. The fields commonly used are produced by large electromagnets and are of the order of 5,000 to 20,000 gauss. On application of the field the sample will, if diamagnetic, seem to lose in weight, or if paramagnetic, seem to gain in weight. The changes so observed are of the order of milligrams.

If the sample is a liquid or a powder, it may be contained in a calibrated glass tube. For most measurements on organic compounds, it is necessary to use a microbalance to gain adequate sensitivity. Temperature control is generally necessary, and for paramagnetic substances it is frequently necessary to make measurements over a wide range of temperature. With all refinements, a sensitivity of four significant figures is attained without too much difficulty.

There have been innumerable modifications of the Gouy method and many other methods operating on somewhat different principles. Thus, the sample may be suspended horizontally rather than vertically, the force may be measured by springs, or by hydrostatic pressure. In all methods related to the Gouy method the force, $f$, exerted on application of the field is given by

$$
f=1 / 2\left(\mathrm{~K}-\mathrm{K}_{0}\right) A H^{2},
$$

where $\mathrm{K}$ is the magnetic susceptibility of the substance per unit volume, $K_{0}$ is the volume susceptibility of the atmosphere surrounding the sample, $A$ is the cross-sectional area of the sample, and $H$ is the field strength. The susceptibility of hydrogen as an atmosphere is generally negligible, but air is appreciably paramagnetic.

The Faraday method for measuring susceptibilities measures the force exerted on a small sample by a field having a fairly high gradient in the direction of motion of the sample. The Quincke method is often used for liquids; it measures the capillary rise, or depression, produced when a strong field is applied to the liquid [1 to 5$]^{1}$.

Some 30 or 40 years ago, efforts were made to apply standard induction techniques to the measurement of magnetic susceptibilities. These methods all failed because the susceptibilities are so very small. But recently Broersma [6] has developed an induction method that is as sensitive as the best Gouy determinations. At present the method is considerably more complicated than the Gouy method, but further development may make it the method of choice for routine susceptibility measurements.

\section{Atomic and Molecular Diamagnetism}

The classical theory of diamagnetism shows that the sưsceptibility per gram atom, $\chi_{A}$, is given by the expression

\footnotetext{
1 Figures in brackets indicate the literature references at the end of this paper.
} 


$$
\chi_{A}=-2.832 \sum r^{\overline{2}} \times 10^{10},
$$

where $\overline{r^{2}}$ is the mean square radius of the electronic orbits. The same result is obtained in the quantum-mechanical theory of magnetism, from which diamagnetic atomic susceptibilities may in theory be directly calculated [7]. For hydrogen

$$
\begin{aligned}
& \chi_{A}=-2.832 \times 10^{10}\left\{\frac{h^{2}}{4 \pi^{2} Z e^{2} m}\right\} \\
& {\left[\frac{5}{2} n^{4}-\frac{3}{2} n^{2} l(l+1)+\frac{1}{2} n^{2}\right]}
\end{aligned}
$$

where $n$ and $l$ are principal and subordinate quantum numbers, respectively, $Z$ is the atomic number, $h$ is Planck's constant, and $m$ is the electron mass. Unfortunately, atomic hydrogen is not only difficult to study, but is paramagnetic. This paramagnetism is far larger than the underlying diamagnetism could possibly be. However, indirect methods for estimating the atomic diamagnetism give results in good agreement with theory. Similar calculations give fair agreement with experiment for the inert gases and for mercury vapor.

Unfortunately, theoretical calculations of susceptibility for polyatomic molecules are in a rudimentary state. For the calculation of molecular susceptibilities our only recourse, at present, is the use of empirical constants derived from the consideration of experimentally found susceptibilities for a large number of organic compounds. These constants, known as Pascal's constants, may be used in much the same way that molecular refractivities are used in calculating molar refraction. The molar susceptibility is in general given by

$$
\chi_{M}=\sum n_{A} \chi_{A}+\lambda
$$

where $n_{A}$ is the number of atoms of susceptibility, $\chi_{A}$ is the molecule, and $\lambda$ is a constitutive correction depending on the nature of the bonds between the atoms. Tables of Pascal's constants and constitutive corrections are available in works on magnetochemistry [2]. With their aid some surprisingly accurate estimations of susceptibilities have been made.

These considerations suggest that the process of polymerization should bring about a change of magnetic susceptibility. Farquharson [8] has examined the polymerization of 2,3-dimethyl- butadiene. Consider a dimerization $2 B \rightarrow B_{2}$. The molar susceptibility of the dimer must be $\chi_{M}=2 \chi_{B}+\lambda$, where $\lambda$ is the constitutive correction for formation of the new bond. If $n$ molecules of $B$ polymerize to form $B_{n}$, then $\chi_{M}=n \chi_{B}+(n-1) \lambda$, and the relationship between molar suceptibility and $n$ will be a straight line. The susceptibility of the polymer per unit mass will be

$$
\chi=\frac{n \chi_{B}+(n-1) \lambda}{n M_{B}},
$$

where $M_{B}$ is the molecular weight of the monomer. The change of $\chi$ with $n$ will then be represented by a hyperbola. Farquharson has found that such is the case for 2,3-dimethylbutadiene, and for certain other polymerizations. Whether the susceptibility increases or decreases during polymerization depends on the nature of the bonds broken and formed. Both cases are known.

From the above it would appear that measurement of magnetic susceptibility may afford a measure of the extent of polymerization and also an estimate of the molecular weight. Applications of the latter have been made by Farquharson [9] to the polyoxymethylenes. So far, however, there is no evidence to show that the magnetic method can compete in these respects with any of the standard procedures in polymer chemistry. It might possibly be adapted to certain in situ types of studies where other methods are awkward. But the small differences of susceptibilities encountered and the effect of isomerism, hydrogen bonding, and impurities, is such as to discourage further attempts along this line.

\section{Diamagnetic Anisotropy}

We turn now to a much more promising application of magnetism to high polymers, namely, the diamagnetic anisotropy.

A substance, such as a gas, in which the molecules are all arranged at random, is truly isotropic. Effective isotropy may be shown by a solid, the microcrystals of which have completely random orientation. In crystals of low symmetry there are three mutually perpendicular directions known as the axes of principal magnetism. Along these axes the direction of magnetism corresponds with the direction of the applied field. The magnetic susceptibilities along these axes are called principal susceptibilities. Their values are often 
unequal. If such an anisotropic substance is powdered, the average susceptibility of the powder is the mean of the three principal susceptibilities,

$$
\bar{\chi}=1 / 3\left(\chi_{1}+\chi_{2}+\chi_{3}\right) .
$$

Cubic crystalline matter is isotropic, but if a piece of noncubic matter is supended in a magnetic field it will tend to orient itself so that the axis of maximum (algebraic) susceptibility in the plane of rotation lies along the lines of force. In orthogonal crystals the axes of principal susceptibility coincide with the crystal axes. In the monoclinic system one principal magnetic axis coincides with the symmetry axis of the crystals. Many organic compounds crystallize in the monoclinic system.

The additivity of average suceptibilities of organic compounds shows that even in the solid state the mutual influence of neighboring molecules on the magnetic susceptibility must be neglibible. (This is not true for paramagnetic compounds.) It follows that the anisotropy of a single crystal must be due to the anisotropy of the unit cell, and that this, in turn, depends only on the resultant anisotropy of the individual molecules in the cell. If the molecule is essentially magnetically isotropic, then the crystal will show little or no anisotropy. But if the molecule is anisotropic then the resultant anisotropy of the crystal depends only on the relative orientation of the molecules. If the molecules are arranged in layers, then the resultant crystal anisotropy will be large, but if the molecules are arranged haphazardly, or so that their anisotropies cancel each other, then the crystal will be isotropic.

An obvious way to measure magnetic anisotropy is to orient a single crystal so that one axis lies in the field gradient in one of the conventional methods for measuring susceptibilities. But Krishnan [10] has developed a far more elegant method. Krishnan's method gives the difference between any two principal susceptibilities.

If an isotropic sample is cut to spherical shape it will suffer no orientation in a nonuniform field. Alternatively, an isotropic sample of any shape will suffer no orientation in a uniform field. This is the basis of the Krishnan method.

The sample is suspended by a fine torsion fiber in a homogeneous field (fig. 2). A field of sufficient homogeneity may be found in a small region

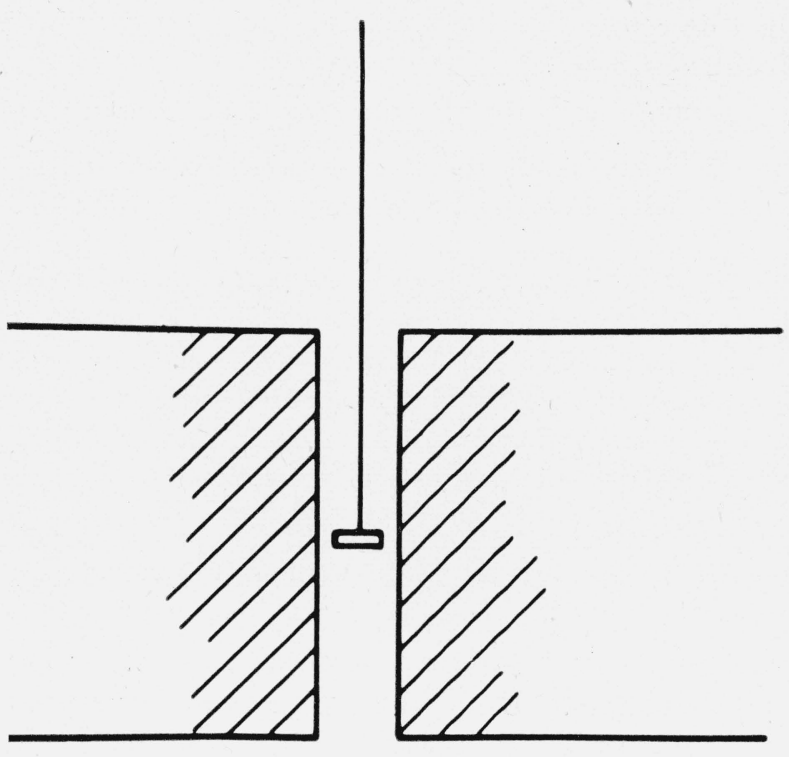

Figure 2. Principle of the Krishnan anisotropy balance.

between relatively large plane pole pieces. The field may be 5,000 to 10,000 gauss. For reasons of economy a permanent magnet, mounted on a sliding table, has some advantages for these measurements. In general one magnetic axis of the sample is in the axis of the torsion fiber. If the crystal is isotropic it will suffer no orientation on application of the field, but if it is anisotropic it will turn until the algebraically largest susceptibility approaches the direction of the lines of force. The torsion head may now be turned until the crystal suffers no orientation on application of the field. The largest algebraic susceptibility is now parallel to the field.

If the crystal is made to oscillate, the period of oscillation is related to the molar magnetic anisotropy as follows:

$$
\Delta \chi=\frac{C}{H^{2}} \frac{M}{m} \frac{t_{0}^{2}-t^{2}}{t^{2}},
$$

where $t_{0}$ and $t$ are oscillation periods with the field off and on, respectively, $C$ is the torsional constant of the fiber, $m$ is the mass of the crystal, $M$ is the molecular (or formula) weight, $H$ is the field, and $\Delta \chi$ is the difference between the two principal susceptibilities in the plane of rotation.

The anisotropy so obtained is that which exists in the plane of oscillation. The third principal susceptibility may be investigated by reorienting the crystal. Absolute principal susceptibilities may be obtained by measuring one susceptibility 
by a direct method; or by using the average (powder) susceptibility.

Krishnan [11] has also developed a modification of the above method. This second procedure has some practical advantages over the first, especially for very small crystals. The crystal orientation in the field is adjusted as before so that the largest algebraic susceptibility in the plane of rotation lies in the direction of the field. Now if the torsion head is turned through an angle $\alpha$, the crystal will turn through a smaller angle $\phi$. The relation between $\alpha$ and $\phi$ is such that

$$
c(\alpha-\phi)=\frac{1}{2} \frac{m}{M} H^{2} \Delta \chi 2 \phi,
$$

where the terms have the same significance as before. If the torsion head rotation is continued until $\phi=\pi / 4$ the crystal will suddenly flip around to a new equilibrium position. For this to occur the torsion head must be turned through an angle $\alpha_{c}$, and we have:

$$
c\left(\alpha_{c}-\pi / 4\right)=\frac{1}{2} \frac{m}{M} H^{2} \Delta \chi,
$$

or

$$
\Delta \chi=\frac{c}{H^{2}} \frac{M}{m}\left(2 \alpha_{c}-\pi / 2\right) .
$$

The largest diamagnetic anisotropies are shown by graphite, and by aromatic compounds, the molecules of which contain benzene, cyanuric, or phthalocyanic rings. Carbon in the cubic (diamond) form is isotropic with a molar susceptibility of $-6 \times 10^{-6}$. But the principal molar susceptibility of graphite parallel to the hexagon axis is $-264 \times 10^{-6}$, which is over forty times numerically as great as the susceptibility normal to the hexagonal axis. For naphthalene and anthracene the principal susceptibilities are given

\begin{tabular}{|c|c|c|c|}
\hline & $-\chi_{1}$ & $-\chi_{2}$ & $-\chi_{3}$ \\
\hline Naphthalene & 56. 0 & 146. 4 & 76. 6 \\
\hline Anthracene $_{\ldots}$ & 75.5 & 211.8 & 102. 9 \\
\hline
\end{tabular}
below [12]:

Principal susceptibilities for single crystal $\times 10^{6}$

For many organic compounds the complete structure has been determined by X-ray diffrac- tion studies, and the orientations of the molecules in the unit cell are known. It is possible to deduce the principal susceptibilities of the individual molecules from the principal susceptibilities of the crystal and the molecular directional cosines. Principal molar susceptibilities for individual molecules of two compounds are shown below.

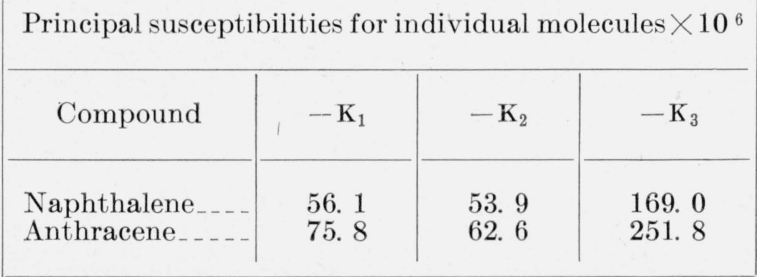

Conversely, if the principal susceptibilities of the individual molecules are known or can be estimated, and if the anisotropy of the crystal is measured, it is then possible in favorable cases to find the arrangement of molecules in the unit cell. In other words, the magnetic anisotropy may be used for a complete structure determination. More frequently, the anisotropy is of use in simplifying structural determinations by X-rays. Examples of these calculations will be given below.

Various suggestions have been made to explain the abnormal diamagnetism shown along one axis by aromatic compounds. The most popular explanation is that the aromatic, or resonance, electrons occupy orbits of molecular instead of atomic size. A satisfactory general theory of anomalous diamagnetic anisotropy in aromatic compounds has been developed by London [13]. London's theory corresponds to the method of molecular orbitals in the theory of chemical binding.

We shall now show how the observed principal susceptibilities in the crystal may be used to find the principal susceptibilities for the individual molecule. Naphthalene is chosen as the example.

Naphthalene belongs to the monoclinic class. The crystalline and magnetic axes of a monoclinic crystal are shown in figure 3 , and figure 4 shows the relation of a naphthalene molecule to these axes. Two of the principal magnetic axes lie in the (010) or $a c$ plane; the magnetic susceptibilities along these axes are $\chi_{1}$ and $\chi_{2}$. The angle $\psi$, which the $\chi_{1}$ axis makes with the $c$ axis of the crystal, taken as positive toward the obtuse angle $\beta$ between the $c$ and $a$ axes, determines the positions of the two magnetic axes. The angle $\theta$ is connected 


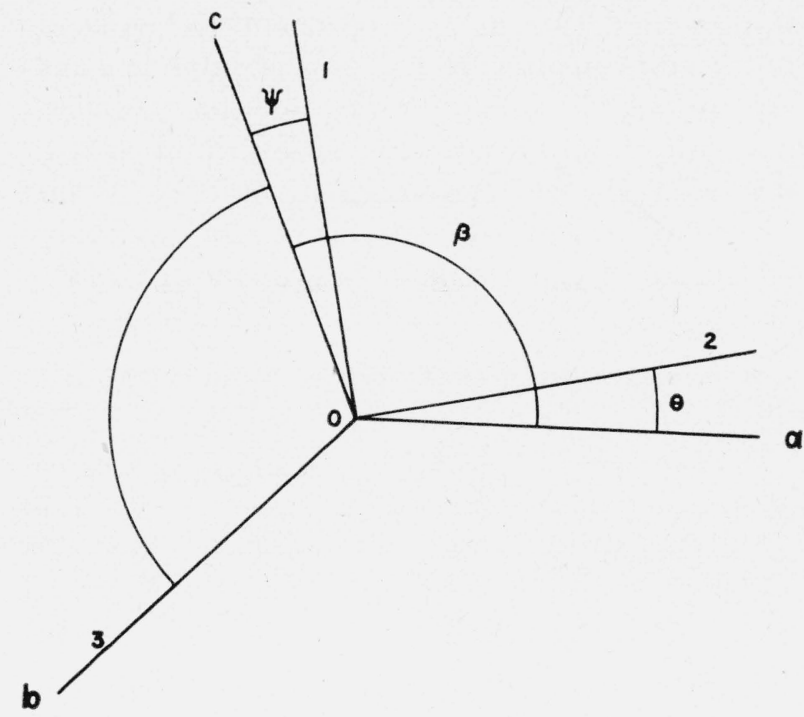

Figure 3. Principal crystal $(a, b, c)$ and magnetic $(1,2,3)$ axes in a monoclinic crystal.

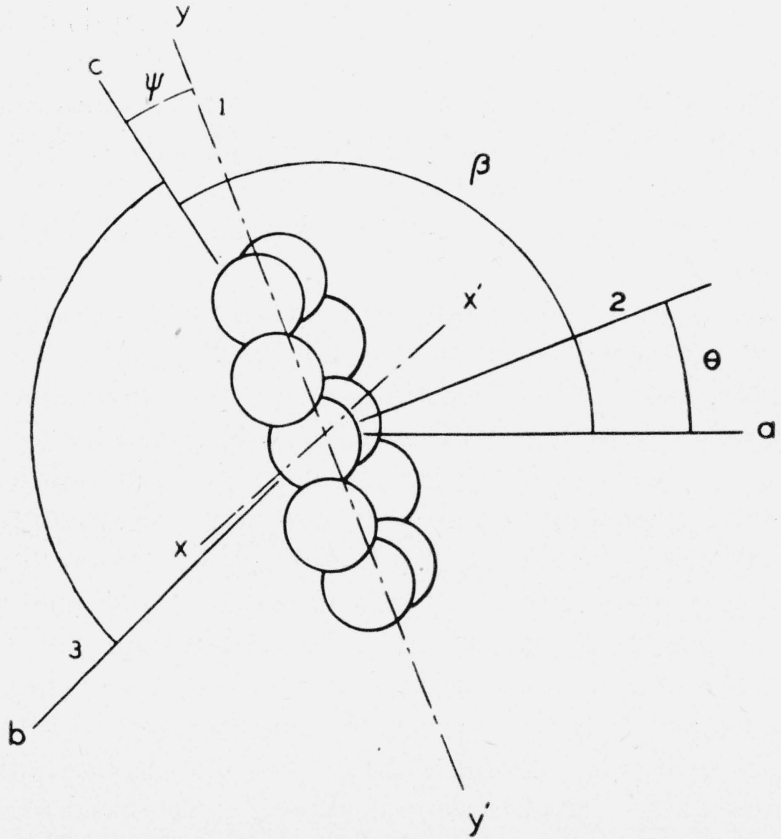

Figure 4. Position of naphthalene molecule relative to crystal and magnetic axes.

with $\psi$ by the relation $90^{\circ}+\theta+\psi=$ obtuse $\beta$. The third magnetic axis is along the $b$ axis, and the susceptibility along it is $\chi_{3}$.

X-ray studies show that the unit cell of naphthalene contains two molecules, for each of which the structure is plane. Both molecules have their lengths, the lines that join centers of constituent rings, almost in the (010) plane, making an angle of $12^{\circ}$ with the $c$ axis. Considerations of sym metry show that the long axis of the naphthalene molecule must be one of its magnetic axis. We should, therefore, expect the above direction in the crystal to be one of the magnetic axes of the crystal. Direct observation of the magnetic axes confirms this view, and the angle $\psi$ is actually found to be $12^{\circ}$. The other two magnetic axes are along the breadth of the molecule in the plane of the rings, and along the normal to the plane of the rings, respectively.

X-ray studies show that the planes of the molecules are inclined at $+65^{\circ}$ and $-65^{\circ}$, respectively, to the (010) plane. We have, therefore, all the information necessary to find the magnetic susceptibilities of the individual molecule

$$
\begin{aligned}
& \chi_{1}=\mathrm{K}_{1}, \\
& \chi_{2}=\mathrm{K}_{2} \cos ^{2} 65^{\circ}+\mathrm{K}_{3} \sin ^{2} 65^{\circ}, \\
& \chi_{3}=\mathrm{K}_{2} \sin ^{2} 65^{\circ}+\mathrm{K}_{3} \cos ^{2} 65^{\circ},
\end{aligned}
$$

where $\chi$ and $K$ are principal molar susceptibilities of crystal and of molecule, respectively. Substituting the experimentally determined values for $\chi_{1}, \chi_{2}, \chi_{3}$, we obtain the numerical values for $\mathrm{K}_{1}, \mathrm{~K}_{2}, \mathrm{~K}_{3}$ given above.

Biphenyl will be chosen as an example to illustrate the type of calculation in which the anisotropy is used to find the orientation of molecules in the unit cell. We shall assume that the rings in biphenyl have the same structure as in benzene and that the molecule is planar.

The average (powder) molar susceptibility of biphenyl is $-102.9 \times 10^{-6}$, which is numerically less than twice that of benzene by $7.7 \times 10^{-6}$. This difference is obviously the contribution of the two hydrogen atoms that have been dropped. As a first approximation, we assume that this diminution is the same along the three principal axes of the molecule. The principal susceptibilities for the benzene molecule are $\mathrm{K}_{1}=-37.3, \mathrm{~K}_{2}=-37.3$ $\mathrm{K}_{3}=-91.2\left(\times 10^{-6}\right)$. We then obtain for the principal molar susceptibilities of the biphenyl molecule, all $\times 10^{-6}$,

$$
\begin{aligned}
\mathrm{K}_{1}=\mathrm{K}_{2} & =-(2 \times 37.3)-7.7=-66.9 \\
\mathrm{~K}_{3} & =-(2 \times 91.2)-7.7=-174.7 .
\end{aligned}
$$

The principal molar susceptibilities for the biphenyl crystal are

$$
\chi_{1}=-63.4 \times 10^{-6}
$$




$$
\begin{aligned}
& \chi_{2}=-146.5 \\
& \chi_{3}=-98.9,
\end{aligned}
$$

and the angle $\psi$ is $20.1^{\circ}$.

There are two molecules in the unit cell. Place both molecules with their planes parallel to (100) and their lengths along the $c$ axis. To bring the molecules to their actual orientations, they are given the following rotations (fig. 5):

First, a rotation about the $c$ axis of one of the molecules through an angle $\lambda$, and of the other through an angle $-\lambda$.

Second, a rotation of both molecules about the $b$ axis through an angle $\delta$, the positive direction of the rotation being defined as from the $c$ axis to the $a$ axis, through the obtuse angle $\beta$.

Third, a rotation of the molecules through $+\nu$ and $-\nu$, respectively, about the normal to the plane that contains the $b$ axis and the direction of lengths of the molecules after the second rotation has been performed.

It is clear that $\delta=\psi=+20.1^{\circ}$, and $\lambda$ and $\nu$ can be obtained from the following relationships:

$$
\begin{aligned}
& \chi_{1}=K_{1} \cos ^{2} \nu+\left(K_{2} \cos ^{2} \lambda+K_{3} \sin ^{2} \lambda\right) \sin ^{2} \nu \\
& \chi_{2}=K_{2} \sin ^{2} \lambda+K_{3} \cos ^{2} \lambda \\
& \chi_{3}=K_{1} \sin ^{2} \nu+\left(K_{2} \cos ^{2} \lambda+K_{3} \sin ^{2} \lambda\right) \cos ^{2} \nu .
\end{aligned}
$$

It has already been assumed that $\chi_{1}+\chi_{2}+\chi_{3}=$ $\mathrm{K}_{1}+\mathrm{K}_{2}+\mathrm{K}_{3}$; therefore, only two of the above relationships are independent.

Solving, we get

$$
\lambda=31^{\circ}, \quad \nu=0^{\circ} .
$$

The lengths of the molecules lie in the (010) plane in the obtuse angle $\beta$ at $20.1^{\circ}$ to the $c$ axis, and the planes of the molecules are inclined at plus and minus $31^{\circ}$, respectively, to the $b$ axis. This structure determination is confirmed by $\mathrm{X}$-ray analysis. $\left(\psi=20^{\circ}, \lambda=32^{\circ}, \nu=0^{\circ}\right)$.

In the above analysis we have evaluated the principal susceptibilities of the molecule from structure considerations that may not be applicable for molecules more complicated than biphenyl. In such cases it is possible to obtain the molecular susceptibilities from measurements of magneto-optical rotation, according to methods described by Raman and Krishnan [14].

The structure determination given for biphenyl

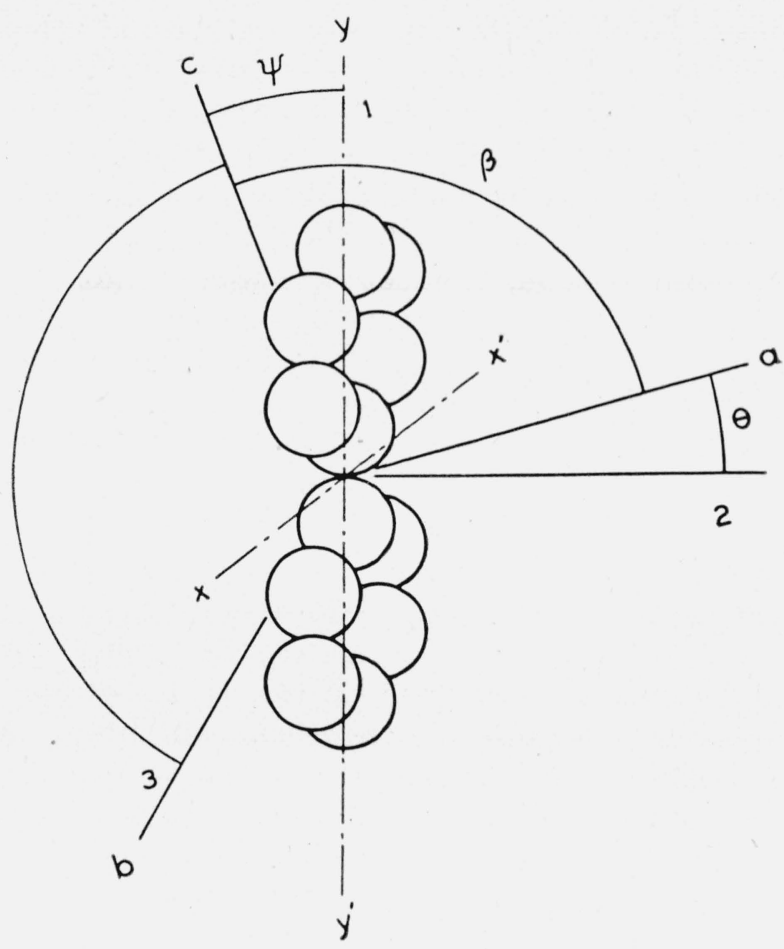

Figure 5. Position of biphenyl molecule relative to crystal and magnetic axes.

is unfortunately not generally applicable to organic solids. The simplicity of the derivation comes from the special case of $\mu \approx v$. For other classes of substances the information obtainable from magnetic anisotropy is often less, but any aid from such a source is welcome if it lessens the labor of complete structure determinations from X-ray data. The applicability of the magnetic method is described by Lonsdale and Krishnan [12]. It may be summarized as follows:

In the triclinic system, magnetic anisotropy measurements give directly the molecular orientations.

In the monoclinic and orthorhombic systems the magnetic method is of aid in structure determinations and is very valuable in a few special cases.

In crystals of high symmetry the magnetic measurements are of little or no use [15].

The literature does not reveal many studies on the magnetic anisotropy of polymeric material. A few studies have been made that show the existence of the property in high polymers. But the obvious applicability of the method in the study of polymers containing aromatic groups seems to have escaped attention.

Mme. Cotton-Feytis [16, 17] has recently dem- 
onstrated the anisotropy of several natural types of fibers and of crude rubber. These substances can, to a first approximation, be considered as uniaxial crystals. The largest numerical susceptibility is sometimes longitudinal, sometimes perpendicular to the long axis. Various types of cellulose (cotton, sisal, etc.), silk fiber, keratin, and collagen all show varying degrees of anisotropy. The magnitude of the anisotropy roughly parallels the degree of molecular orientation as revealed by X-ray studies. For example, a normal sample of collagen showing a high degree of molecular orientation has an anisotropy ten times as large as the same sample shrunk and rendered amorphous by treatment with formaldehyde.

Nilakantan has investigated the anisotropy of wood and cellulose. Wood is anisotropic, apparently because of a molecular anisotropy in the cellulose molecule and the more or less regular orientation of these molecules along the fiber axis. Lignin and the hemicelluloses are amorphous.

The greatest diamagnetism of cellulose is along the fiber axis, which is presumably along the length of the molecule. This anisotropy in $\alpha$-cellulose derived from teakwood is about $\chi_{\|}-\chi_{\perp}=-0.1 \times$ $10^{-6}$. The average susceptibility of $\alpha$-cellulose in powder form is $-0.508 \times 10^{-6}$. The method appears to have some utility in confirming the molecular orientations of cellulose shown by X-ray studies.

Crude rubber normally has a certain degree of anisotropy, and this is altered by hot and cold working, and by compression and tension. Plexiglass is said by Cotton-Feytis to yield somewhat similar results, but no details are given.

When crude rubber is stretched, the anisotropy increases but seems to tend toward a limit. This parallels the effects observed with X-ray methods and suggests more or less complete molecular orientation in one direction at high elongations. The anisotropy observed in stretched rubber is, incidentally, quite large. The figures reported by Cotton-Feytis for oscillation periods with the magnetic field off and on, respectively, are 85 seconds and about 5 seconds. There is no doubt that the method is a sensitive one for detecting molecular orientation in such systems.

The effects observed will doubtless be much more valuable in the study of high polymers containing aromatic groups. It seems probable that degrees of molecular orientation quite beyond detection by other methods will be readily estimated by the anisotropy measurements. There are obvious applications of such studies to changes occurring under compression, tension, extrusion, and hot and cold working. The establishment of relationships between anisotropy and physical properties such as hardness, tensile strength, optical properties, and second-order transitions, is also a possibility.

\section{Atomic and Molecular Paramagnetism}

We turn now to applications in which the paramagnetism of certain molecules or groups may be used for the elucidation of certain problems in polymerization. First, however, we shall present some general information on atomic and molecular paramagnetism.

The classical theory of Langevin develops an expression for paramagnetic susceptibility on the assumption that each atom is a small permanent magnet. These atomic magnets tend to aline themselves with an applied field, but the alinement is resisted by thermal agitation. In modern terminology we identify the atomic magnets with the magnetic moments produced by orbital and spin movements of the electrons.

Langevin's expression for the molar paramagnetism is

$$
X_{M}=\frac{N \mu^{2}}{3 k T}
$$

where $N$ is Avogadro's number, $\mu$ is the permanent magnetic moment, $k$ the Boltzmann constant, and $T$ the absolute temperature. The corresponding quantum mechanical expression derived by Van Vleck [7] is

$$
X_{M}=\frac{N \overline{\bar{\mu}}^{2}}{3 k T}+N \bar{\alpha},
$$

where $\overline{\bar{\mu}}^{2}$ is the square of the low frequency part of the magnetic moment vector, averaged over time, and this average being itself average over the various normal states appropriately weighted according to the Boltzmann factor. The quantity $\bar{\alpha}$ is a combination of high frequency elements of the magnetic moment, and of the diamagnetic part of the susceptibility.

In general, the paramagnetic moment consists of a part derived from an orbital contribution and a part derived from a spin contribution. The only major cases in which the orbital contribution 
is important is for isolated paramagnetic atoms and for rare earth ions. For those substances in which only the spin contribution is important the paramagnetism is approximately represented by

$$
X_{M}=\frac{N \beta^{2}}{3 k T}[4 S(S+1)],
$$

where $S$ is the resultant spin moment, and $\beta$ is the Bohr magneton, which is given by

$$
\beta=\frac{e h}{4 m c}=0.917 \times 10^{-20} \mathrm{erg}_{\mathrm{gauss}}{ }^{-1},
$$

where $e$ is the electronic charge, $h$ is Planck's constant, $m$ is the mass of the electron, and $c$ is the velocity of light.

The effective magnetic moment in such cases is given by

$$
\mu_{\text {eff }}=2 \sqrt{S(S+1)},
$$

where $S$ may be found from the spectral multiplicity, or, if the number, $n$, of unpaired electrons is known,

$$
\mu_{\text {eff }}=\sqrt{n(n+2)} .
$$

Finally, the magnetic moment may be found experimentally from

$$
\mu_{\text {eff }}=2.84 \sqrt{\chi_{M} T},
$$

or more accurately from

$$
\mu=2.84 \sqrt{\chi_{M}(T+\Delta)} .
$$

These expressions are of great value in connection with studies of transition group ions, such as $\mathrm{Fe}^{+3}, \mathrm{Cr}^{+3}, \mathrm{Ni}^{+2}$, and their respective complexes. However, for all but a very few cases of paramagnetism in molecules there is only one unpaired electron, and the "spin only" formula applies. Furthermore, in almost all such cases the quantity $\triangle$ is not far from zero. Hence, for paramagnetic molecules such as triphenylmethyl, we find that the molar paramagnetic susceptibility at $20^{\circ} \mathrm{C}$ is about $1,270 \times 10^{-6}$, and that this varies inversely as the absolute temperature.

Among paramagnetic molecules of interest we may mention $\mathrm{NO}, \mathrm{NO}_{2}, \mathrm{ClO}_{3}$, the triarylmethyls, semiquinones, and metal ketyls. All these substances are characterized by having an odd number of electrons. The existence of an unpaired electron is essential for paramagnetism. Molecular oxygen is paramagnetic, although it contains an even number of electrons. The paramagnetism in this case results from the peculiar electronic state in which two electrons remain unpaired. A similar situation exists in a few complex organic compounds related to the Chichibabin hydrocarbons. Such substances are called biradicals.

It will be clear from the above that a major application of magnetochemistry is in the detection and estimation of molecules containing an unpaired electron spin, that is to say, of free radicals.

\section{Free Radicals}

There have been many magnetochemical studies of free radicals reported in the past 10 years, during which this has become the leading physical method in free-radical chemistry. The general nature of the results obtainable will be surveyed briefly $[2,19]$.

Inasmuch as the most characteristic property of a free radical is its unpaired electron, the magnetic susceptibility is a most direct measure of free radical existence. Whereas molecular weights, colors, and chemical reactivity may depend upon secondary factors, it is difficult to see how a molecule that does not contain a transition group element can be paramagnetic unless it is a free radical. We choose to define a free radical as a chemical entity, neutral or ionic, which contains one or more unpaired electrons, transition elements being, of course, excluded.

In this way the free radical nature has been established for such substances as diaryl nitric oxide, Fremy's salt, a great variety of hexaarylethanes, the hydrazyls, semiquinones, Wurster's salts, the metal ketyls, certain diaryl peroxides, diaryl disulfides, and of certain materials in the phosphorescent state. On the other hand, certain organometallic compounds of which hexaphenyldilead is an example, have been shown to be diamagnetic, although molecular weight determinations seem to indicate dissociation to the free radical form.

The magnetic method has a further advantage in its flexibility. Measurements may conveniently be made on free radicals in solution at various temperatures and concentrations, and in various solvents. The importance of this type of measurement lies in the possibility of obtaining equilibrium constants at several temperatures, and thus 
opening the way for calculations of heats of dissociation, free energies, and entropies. A considerable number of hexaarylethanes have been studied in this way with a view to finding the effect of different substituents on the heats of dissociation. Such data are of interest in connection with the resonance theory of free radical stability.

Other information obtainable from magnetic measurements on hexaarylethanes includes the rate and activation energy of the disproportionation reaction undergone by some free radicals, the relationships between color and free radical concentration, and rate studies on certain photochemical reactions shown by these substances. One of the most valuable features of the magnetic method is the possibility of studying, in situ, reactions as they take place, provided the concentration of a paramagnetic component changes with time, as is the case with free radical reactions [21, 22].

It should be mentioned that the magnetic method is not infallible. Short-lived radicals cannot be studied with the magnet unless the steady state concentration is sufficiently high. With the most refined apparatus a free radical concentration of $10^{-6}$ mole per liter might be just detectable. Furthermore, there is one case reported in the literature in which the substance appears to be a biradical but is diamagnetic. This is the simple Chichibabin hydrocarbon, to derivatives of which reference was made above. The simple hydrocarbon appears to catalyze the ortho-para hydrogen conversion, which is evidence of a paramagnetic component, but the compound shows no trace of paramagnetism as measured in the usual way [23].

\section{Magnetic Measurements on Reacting Systems}

As applied to reacting systems, magnetic susceptibilities have been used as described above to study the rate of disproportionation of hexaarylethanes [24]. The transitory existence of semiquinone free radicals has been demonstrated magnetically by Michaelis [25], who studied the concentration of these substances as a function of time, and in some cases by a process that might be called "magnetic titration," that is, the change of susceptibility during the quantitative addition of an oxidizing or reducing agent. A typical example of a reaction that may be studied in situ magnetically is the slow reduction of dichromate with glucose in dilute solution. This reaction produces a large calculable change of susceptibility owing to the change from the practically nonmagnetic dichromate ion to the strongly paramagnetic chromic ion. No change of phase complicates this reaction, which has been used to calibrate the very sensitive magnetic balance described below.

The possibility of studying reacting systems in situ has suggested the use of magnetic measurements in polymerization reactions. The information obtainable in this way would appear to be of two kinds. First, it would certainly be possible to detect very small concentrations of molecular oxygen and to estimate their changes with time. And second, the free radical theory of polymerization suggests the attractive possibility of obtaining quantitative information on free radical concentration during polymerization.

The magnetic study of reactions such as these requires a degree of sensitivity far beyond that normally obtainable with the classical Gouy balance. An apparatus using the Gouy principle, but with several refinements leading to greatly increased sensitivity, will be described (fig. 6). This

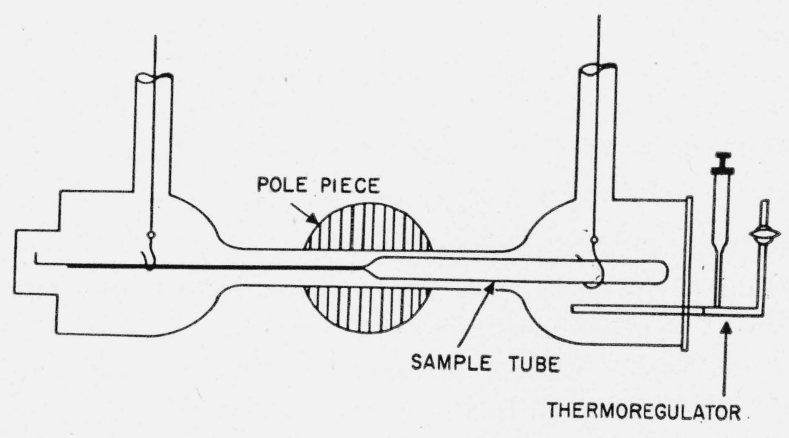

Figure 6. Horizontal Gouy balance.

uses a horizontal, rather than a vertical, mounting of the sample tube. The horizontal mounting has actually been used for many years, and has recently been adopted by Theorell and by Calvin for high sensitivity measurements.

The sample tube is suspended horizontally from long delicate fibers. One end of the tube is between the poles of a magnet. A permanent magnet has a great advantage in that the field is quite steady over long periods of time. This is a condition very difficult to attain with an electromagnet. The substance under investigation is 
placed in the sample tube, which then moves horizontally as the susceptibility increases or decreases with time as the reaction proceeds. The whole apparatus is, of course, mounted so as to reduce the effects of vibration and of drafts to a minimum.

Displacements of the sample tube are observed for approximate readings with the aid of a micrometer microscope focussed on a fiduciary point. Refined measurements are made with a Fabry and Perot type of interferometer, one plate of which is mounted on the end of the sample tube. With this apparatus the sensitivity is such that $10^{-6}$ mole of free radicals may just be detected [26].

Some of the results obtained with this apparatus are shown in figure 7 . These results are for the

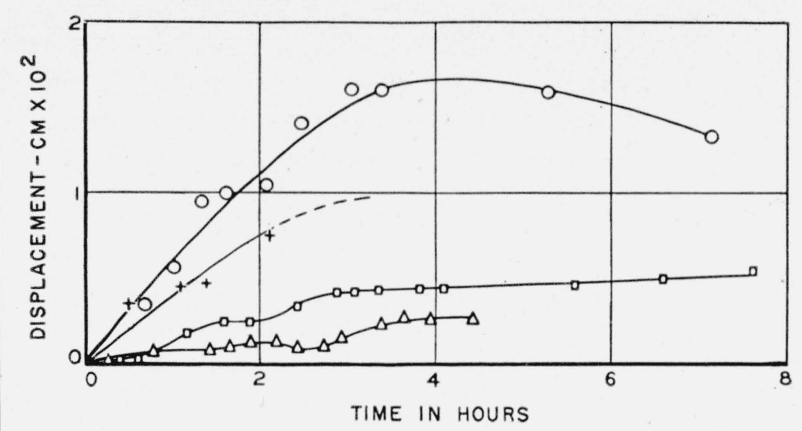

Figure 7. Changes of diamagnetic susceptibility during polymerization of styrene.

Thermal polymerization of styrene. $O$, stock room styrene, vacuum distilled (+ degassed); $\square$, styrene prepared from cinnamic acid, and $\Delta$, degassed.

thermal polymerization of styrene. It will be noted that the magnetic susceptibility suffers relatively large changes during the first few hours of reaction. Under the experimental conditions this corresponds to a few percent of polymerization. The changes in susceptibility generally take the form of a large increase in diamagnetism followed by a much slower increase. The changes are markedly influenced by the purity of the styrene and the method of handling the monomer prior to its introduction into the apparatus.

The initial increase of diamagnetism is often much greater than would be found for complete polymerization. This "anomalous" diamagnetic increase is believed to be due to the consumption of dissolved molecular oxygen. The inhibiting action of oxygen on vinyl polymerization is well known. Assuming that no normal polymerization takes place during the induction period (a small amount of polymerization would have a negligible effect on the susceptibility), then the displacement of the sample tube from zero time to the time at which the curve levels out, approximately represents the initial concentration of oxygen present. For curve 1 , figure 7 , the initial concentration of oxygen thus found is $2.9 \times 10^{-4}$ mole per liter, and this concentration steadily falls. At 2 hours the concentration is $0.99 \times 10^{-4}$ mole per liter.

The rate of oxygen consumption is shown by these results to be zero order. Reasonably concordant results are obtained on different samples of styrene, although the initial oxygen concentration may be quite different.

These results may be used to calculate the rate constant for initiation. If the initiation process is represented as forming a biradical, oxygen reacts with these free radicals very rapidly.

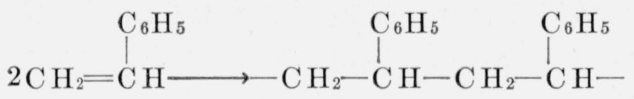<smiles>CCCCC(CC)CC(CC)OCC1COOC(CC)C1</smiles>

Such a process is represented by the equations

$$
\begin{gathered}
2 \mathrm{M} \stackrel{k_{1}}{\longrightarrow} \mathrm{R} \\
\mathrm{R}+\mathrm{O}_{2} \stackrel{k_{2}}{\longrightarrow} \mathrm{RO}_{2},
\end{gathered}
$$

where $R$ is a free radical and represents the concentration of free radicals in the kinetic equations and $M$ is the monomer and its concentration.

$$
\begin{gathered}
\frac{d R}{d t}=K_{1} M^{2}-k_{2}\left[\mathrm{O}_{2}\right] R \\
-\frac{d\left[\mathrm{O}_{2}\right]}{d t}=k_{2}\left[\mathrm{O}_{2}\right] R .
\end{gathered}
$$

Since the rate of consumption of oxygen is experimentally zero order, it follows that eq 1 is the rate-determining step. That is, the free radicals are consumed as rapidly as they are formed owing to the rapid action of the oxygen. A steady state is therefore reached, free radicals being used up 
at the same rate at which they are formed and $d R / d t=0$, therefore

$$
k_{1} M^{2}=K_{2}\left[\mathrm{O}_{2}\right]=-\frac{d\left[\mathrm{O}_{2}\right]}{d t}
$$

and

$$
k_{1}=-\frac{d\left[\mathrm{O}_{2}\right]}{d t} \frac{1}{M^{2}} .
$$

$-d\left[\mathrm{O}_{2}\right] / d t$ may be found from the rate of oxygen loss, and is equal in one of the runs to $2.8 \times 10^{-8}$ mole per liter per second, and $M$ (for pure styrene monomer) is 8.56 moles per liter.

Hence

$$
\begin{aligned}
k_{1} & =2.8 \times 10^{-8} \times 1 /(8.56)^{2} \\
& =3.8 \times 10^{-14} \text { mole per liter per sec. }
\end{aligned}
$$

This figure is not unreasonable. Bartlett [27] gives a rate constant for initiation in the photopolymerization of vinyl acetate (which should be much higher than for thermal polymerization at $66^{\circ} \mathrm{C}$ ) as $\sim 3 \times 10^{-8}$.

The above results are preliminary and are given to indicate the probable direction of magnetochemical research on polymerization rather than with the idea of presenting a completed program.

If vinyl polymerization actually goes through a free radical mechanism there is presented the attractive possibility of determining the free radical concentration by magnetic measurements on reacting systems. There have been some reports in which this possibility is claimed to have been realized [28]. However, the best estimates of free radical concentrations give values of the order of $10^{-9}$ moles per liter. These estimates are made indirectly and are possibly subject to fairly large error. Nevertheless, opinion at the present time is that the sensitivity of the best magnetic balances is still several orders from the detection of free radicals in this way. It may be that an appropriate choice of reacting system would yield free radical concentration much higher than that normally believed to be present. But it must be admitted that such a possibility seems to be somewhat remote.

\section{References}

[1] E. C. Stoner, Magnetism and matter (Methuen and Co., Ltd., London, 1934).

[2] P. W. Selwood, Magnetochemistry (Interscience Publishers, Inc., New York, N. Y., 1943).

[3] W. Klemm, Magnetochemie (Akademische Verlagsgesellchaft, Leipzig, 1936).

[4] S. S. Bhatnagar and R. N. Mathur, Physical principles and applications of magnetochemistry (Macmillan and Co., Ltd., London, 1935).

[5] R. W. Asmussen, Magnetokemiske Unders $\varnothing$ gelser over Uorganiske Kompleksforbindelser (Jul. Gjellurups Forlag, Copenhagen, 1944).

[6] S. Broersma, Magnetic measurements on organic compounds (Martinus Nijhoff, The Hague, 1947).

[7] J. H. Van Vleck, The theory of electric and magnetic susceptibilities (Oxford University Press, Oxford, 1932).

[8] J. Farquharson, Trans. Faraday Soc. 32, 219 (1936).

[9] J. Farquharson, Trans. Faraday Soc. 33, 824 (1937).

[10] K. S. Krishnan, B. C. Guha, and S. Banerjee, Phil. Trans. Roy. Soc. (London) [A] 231, 235 (1933).

[11] K. S. Krishnan and S. Banerjee, Phil. Trans. Roy. Soc. (London) [A]234, 265 (1935).

[12] K. Lonsdale and K. S. Krishnan, Proc. Roy. Soc. (London) [A]156, 597 (1936). This paper corrects certain erroneous data given in one of Krishnan's earlier papers. The incorrect data were unfortunately repeated in a review article by $\mathrm{P}$. W. Selwood and J. Parodi, J. Chem. Education 23, 574 (1946).

[13] F. W. London, J. phys. radium 8, 397 (1937).

[14] C. V. Raman and K. S. Krishnan, Proc. Roy. Soc. [A]113, 511 (1927).

[15] W. A. Wooster, Physics of crystals (Cambridge University Press, Cambridge, Eng.).

[16] E. Cotton-Feytis, Compt. rend. 214, 485, 996 (1942); 215, 299 (1942).

[17] E. Cotton-Feytis, Rev. gen. Caoutchouc 21, 26 (1944).

[18] P. Nilakantan, Proc. Indian Acad. Sci. 7A, 38 (1938).

[19] W. A. Waters, The chemistry of free radicals (Oxford University Press, Oxford, 1946).

[20] R. F. Preckel and P. W. Selwood, J. Am. Chem. Soc. 63, 3,397 (1941).

[21] M. F. Roy and C. S. Marvel, J. Am. Chem. Soc. 59, 2,622 (1937).

[22] H. G. Müller, Z. Elektrochem. 45, 593 (1939).

[23] G. M. Schwab and N. Agliardi, Ber. 73B, 95 (1940).

[24] P. W. Selwood and R. F. Preckel, J. Am. Chem. Soc. 65, 895 (1943).

[25] L. Michaelis, G. F. Boeker, and R. K. Reber, J. Am. Chem. Soc. 60, 202 (1938).

[26] H. Boardman and P. W. Selwood, not yet published.

[27] P. O. Bartlett, J. Am. Chem. Soc. 68, 2,381 (1946).

[28] J. Farquharson and P. Ady, Nature 143, 1,067 (1939). 